\title{
Information Architecture with IBM Task Modeler
}

\author{
Colin Bird \\ IBM United Kingdom Limited \\ Hursley Park \\ Winchester SO21 2JN \\ +44-1962-816025 \\ colinl_bird@uk.ibm.com
}

\author{
Mark Farmer \\ IBM United Kingdom Limited \\ Birmingham Road \\ Warwick CV34 5JL \\ +44-1926-465232 \\ farmerm@uk.ibm.com
}

\begin{abstract}
The IBM Task Modeler supports the rapid creation and analysis of hierarchical task models, thereby providing a valuable and naturally visual tool for information architects. Task Modeler not only facilitates the essential processes of design, validation, and modification but also enables an information architect to develop and apply schemes for information classification.
\end{abstract}

\section{Categories and Subject Descriptors}

H.5.2 [Information Interfaces and Presentation (e.g., HCI)]: User Interfaces - Graphical user interfaces (GUI), Interaction styles. D.2.10 [Software Engineering]: Design Tools and Techniques - Evolutionary prototyping.

\section{General Terms}

Documentation, Design, Experimentation, Human Factors.

\section{Keywords}

Modelling, Task Analysis, DITA, Visualization.

\section{INTRODUCTION}

On the alphaWorks web site, the overview describes Task Modeler as a "tool for modeling human activity as a hierarchy of tasks and related elements.” [1] In our accompanying paper, we demonstrate how Task Modeler enables analysts to develop their task model representations visually and rapidly create, explore, analyse, and share these models [2]. One can fairly easily transform the alphaWorks description to a "tool for information architecture." Information architects work from goal-oriented models of the way in which users will interact with the content, so Task Modeler provides a naturally visual medium for realizing the architectural design.

The information provided by IBM about its solutions and products includes Eclipse-based information centers, for which DITA (the Darwin Information Typing Architecture [3]) is used to author the content as topics and the navigation scheme as a set of maps.

In this paper we consider how the design process can be assisted by visual representation with Task Modeler. Our context for this study is a repository of guidelines and best practices, presented as an information center for use by the Information Development groups at IBM Hursley.

(c) Colin Bird \& Mark Farmer, 2007.

Published by the British Computer Society Volume 2 Proceedings of the 21st BCS HCI Group Conference HCI 2007, 3-7 September 2007, Lancaster University, UK

Devina Ramduny-Ellis \& Dorothy Rachovides (Editors)

\subsection{The information repository}

The basic aim of this repository can be stated as "to encourage commonality between groups by sharing guidance about good practice." The interface to the repository is an Eclipse-based information center, with the content being authored in DITA.

\subsection{Modelling DITA}

Task Modeler uses the hierarchical task analysis (HTA) approach that models human activity as a hierarchy of goals, tasks, and subtasks. Originally developed for the conceptual design of interactive systems, Task Modeler has evolved to comprise several different dialects. The dialect that we shall be considering uses the DITA Modelling perspective. We follow the basic approach described by Larner in his tutorial [4].

\section{MODELLING THE REPOSITORY ARCHITECTURE}

The use model for the information repository envisages members of the information development groups wanting to find out and/or understand aspects of practice related to procedure, policy, and the infrastructure.

Task Modeler provides a naturally visual medium so, in the early stages of representing this use model as an information architecture, we adopt the radial diagram view to capture the essential components without implying any particular sequencing. Our initial view, as shown in Figure 1, comprises a conceptual description of the organisation and its roles and responsibilities, and three high-level ('container') tasks:

- Understanding the tools and technologies used in User Technologies

- Maintaining this repository

- Delivering consistent information

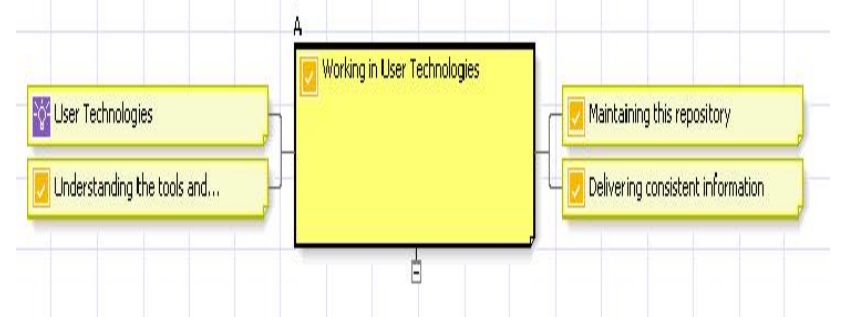

Figure 1, The radial diagram view of the initial representation of the repository use model 
Working with this radial diagram, we decide to alter the initial sequence and to expand the 'Delivering' container task. At this stage, we change to the Indented List view, because its format most closely corresponds to the structure of the DITA map that will be the formal representation of our architecture. Figure 2 shows the Indented List view of the architecture at this stage. Tasks 3 through 6 replace "Delivering consistent information”.

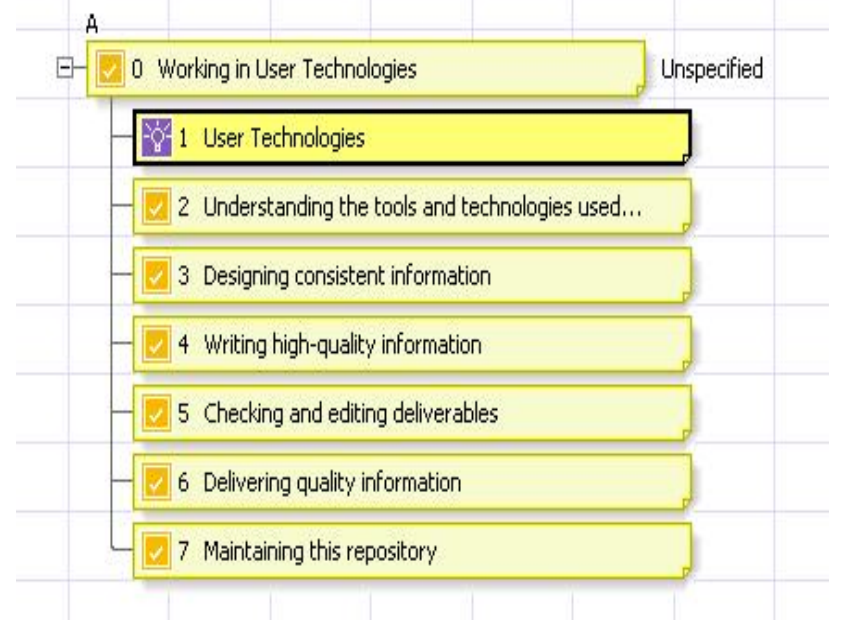

Figure 2. The indented list view of the evolving information architecture

We then evolve the architecture in Task Modeler by creating nodes of different types to reflect the kind of information required to describe the tasks derived from the use model.

\section{TASK MODELER FACILITIES FOR INFORMATION ARCHITECTURE}

In addition to the options for working with DITA maps and topics that Larner describes in [4], Task Modeler provides the following facilities:

- Creating a new information center. Moreover, the next version of Task Modeler will enable the table of contents to be edited and the resulting changes to be written back to the source DITA maps.
- Assembling information components into solutions with the use of DITA anchor and navref elements. Henry \& Fell describe the use of these elements in [5]

- Creating a new map from a template that embodies information architecture best practices

- Visualizing property values, particularly the 'Href status' property

- $\quad$ Visualizing the links in relationship tables

\section{TASK MODELER AS A TOOL FOR INFORMATION CLASSIFICATION}

DITA enables topics and topic collections to be classified with the taxonomy specialization [3, 6]. A fundamental aspect of the classification process is the design, construction, and maintenance of the taxonomy. The DITA Modelling perspective and the hierarchical nature of the models created make Task Modeler an ideal vehicle for working with taxonomies in DITA.

The next version of Task Modeler is planned to include support for the DITA taxonomy specialization. In this paper we shall be presenting and illustrating early results from work on taxonomies for use with information centers sourced with DITA.

\section{REFERENCES}

[1] IBM Task Modeler Overview, http://www.alphaworks.ibm.com/tech/taskmodeler

[2] Farmer, M. and Bird, C, Creating and Analysing Models in IBM Task Modeler, HCI 2007

[3] DITA (Darwin Information Typing Architecture): http://dita.xml.org/

[4] Larner, L., ISTC Communicator, Autumn 2006, pp.14-17

[5] Henry, C. and Fell, J. Best Practices, Volume 8, Issue 5, October 2006

[6] Hennum, E., Anderson, R. and Bird, C. Subject classification with DITA and SKOS, http://www128.ibm.com/developerworks/xml/library/x-dita10/ 